\title{
Stability Analysis of a Flutter Panel with Axial Excitations
}

\author{
Meng Peng and Hans A. DeSmidt \\ Department of Mechanical, Aerospace and Biomedical Engineering, The University of Tennessee, 606 Dougherty Engineering Building, \\ Knoxville, TN 37996-2210, USA \\ Correspondence should be addressed to Meng Peng; mpeng1@vols.utk.edu
}

Received 6 April 2016; Revised 23 June 2016; Accepted 8 July 2016

Academic Editor: Marc Thomas

Copyright ( 2016 M. Peng and H. A. DeSmidt. This is an open access article distributed under the Creative Commons Attribution License, which permits unrestricted use, distribution, and reproduction in any medium, provided the original work is properly cited.

This paper investigates the parametric instability of a panel (beam) under high speed air flows and axial excitations. The idea is to affect out-of-plane vibrations and aerodynamic loads by in-plane excitations. The periodic axial excitation introduces time-varying items into the panel system. The numerical method based on Floquet theory and the perturbation method are utilized to solve the Mathieu-Hill equations. The system stability with respect to air/panel density ratio, dynamic pressure ratio, and excitation frequency are explored. The results indicate that panel flutter can be suppressed by the axial excitations with proper parameter combinations.

\section{Introduction}

Panel (beam) flutter usually occurs when high speed objects move in the atmosphere, such as flight wings [1] and ballute [2]. This phenomenon is a self-excited oscillation due to the coupling of aerodynamic load and out-of-plane vibration. Since flutter can cause system instability and material fatigue, many scholars have carried out theoretical and experimental analyses on this topic. Nelson and Cunningham [3] investigated flutter of flat panels exposed to a supersonic flow. Their model is based on small-deflection plate theory and linearized flow theory, and the stability boundary is determined after decoupling the system equations by Galerkin's method. Olson [4] applied finite element method to the twodimensional panel flutter. A simply supported panel was calculated and an extremely accuracy approximation could be obtained using only a few elements. Parks [5] utilized Lyapunov technique to solve a two-dimensional panel flutter problem and used piston theory to calculate aerodynamic load. The results gave a valuable sufficient stability criterion. Dugundji [6] examined characteristics of panel flutter at high supersonic Mach numbers and clarified the effects of damping, edge conditions, traveling, and standing waves. The panel, Dugundji considered, is a flat rectangular one, simply supported on all four edges, and undergoes two-dimensional midplane compressive forces.
Dowell $[7,8]$ explored plate flutter in nonlinear area by employing Von Karman's large deflection plate theory. Zhou et al. [9] built a nonlinear model for the panel flutter via finite element method, including linear embedded piezoelectric layers. The optimal control approach for the linearized model was presented. Gee [10] discussed the continuation method, as an alternate numerical method that complements direct numerical integration, for the nonlinear panel flutter. Tizzi [11] researched the influence of nonlinear forces on flutter beam. In most cases, the internal force in panel or beam results from either external constant loads or geometric nonlinearities. Therefore, their models are time-invariant system.

Panel is usually excited by in-plane loads resulting from the vibrations generated and/or transmitted through the attached structures and dynamics components when experiencing aerodynamic loads. If the in-plane load is time dependent, the system becomes time-varying. The topic of dynamic stability of time-varying systems attracts many attentions. Iwatsubo et al. [12] surveyed parametric instability of columns under periodic axial loads for different boundary conditions. They used Hsu's results [13] to determine stability conditions and discussed the damping effect on combination resonances. Sinha [14] and Sahu and Datta $[15,16]$ studied the similar problem for Timoshenko beam and curved panel, respectively, and both models are classified as Mathieu-Hill 


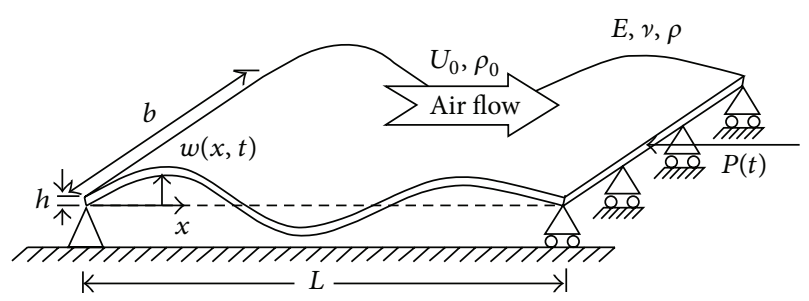

FIGURE 1: Simply supported panel (beam) subjected to an air flow and an axial excitation.

equations. Furthermore, the stability of the nonlinear elastic plate subjected to a periodic in-plane load was analyzed by Ganapathi et al. [17]. They solved nonlinear governing equations by using the Newmark integration scheme coupled with a modified Newton-Raphson iteration procedure. In addition, many papers have been published for dynamic stability analyses of shells under periodic loads [18-24]. Furthermore, Hagedorn and Koval [25] considered the effect of longitudinal vibrations and the space distributed internal force. The combination resonance was analyzed for Bernoulli-Euler and Timoshenko beams under the spatiotemporal force. Yang et al. [26] developed a vibration suppression scheme for an axially moving string under a spatiotemporally varying tension. Lyapunov method was employed to design robust boundary control laws, but the effect of parameters of the spatiotemporally varying tension on system stability has not been fully analyzed.

Nevertheless, the published investigations on the parametric stability of the flutter panel (beam) with the periodically time-varying system stiffness due to axial excitations are scarce. This paper is to explore the coactions of time-varying axial excitations and aerodynamic loads on panel (beam) and conduct parameter studies. The stability analysis is executed first by Floquet theory numerically and then by Hsu's method analytically for approximations.

\section{System Description and Model}

The configuration considered herein is an isotropic thin panel (beam) with constant thickness and cross section. As shown in Figure 1, the panel is simply supported at both ends and a periodic axial excitation acts on the right end. The panel's upper surface is exposed to a supersonic flow, while the air beneath the lower surface is assumed not to affect the panel dynamics. Another assumption made here is the axial strain from the lateral displacement is very small so that it can be ignored. The system model is based on the coupled effects from the out-of-plane (lateral) displacement of the panel, aerodynamic loads, and time-varying in-plane (axial) excitation forces.

The total kinetic energy of the penal due to lateral displacements is

$$
T=\frac{1}{2} \rho A \int_{0}^{L} \dot{w}(x, t)^{2} d x,
$$

where $w(x, t)$ is the lateral displacement of the panel measured in the ground fixed coordinate frame, $\rho$ the panel density, $A$ the panel cross-sectional area, and "." the differentiation with respect to time $t$.

The total potential energy of the panel due to lateral displacements is

$$
V=\frac{1}{2} E^{*} I \int_{0}^{L} w^{\prime \prime}(x, t)^{2} d x+\frac{1}{2} P(t) \int_{0}^{L} w^{\prime}(x, t)^{2} d x,
$$

where $E^{*}=E /\left(1-v^{2}\right)$ for panel and $E^{*}=E$ for beam with Young's modulus $E$ and Poisson's ratio $v$; the moment of inertia is given by $I=b h^{3} / 12$ with panel width $b$ and panel thickness $h ; P(t)$ is the periodic axial excitation with the frequency $\omega$ and " $l$ " indicates differentiation with respect to axial position $x$.

For material viscous damping, a Rayleigh dissipation function is defined as

$$
R=\frac{1}{2} \xi E^{*} I \int_{0}^{L} \dot{w}^{\prime \prime}(x, t)^{2} d x
$$

Here, $\xi$ is the material viscous loss factor of the panel.

The aerodynamic load is expressed by using the classic quasisteady first-order piston theory $[4,6,7,9,11]$ :

$$
p(x, t)=\frac{2 q_{0}}{\beta}\left[\frac{\partial w(x, t)}{\partial x}+\frac{\left(\mathrm{Ma}^{2}-2\right)}{\left(\mathrm{Ma}^{2}-1\right)} \frac{1}{U_{0}} \frac{\partial w(x, t)}{\partial t}\right],
$$

where $q_{0}=\rho_{0} U_{0}^{2} / 2$ is the dynamic pressure, $\rho_{0}$ is the undisturbed air flow density, $U_{0}$ is the flow speed at infinity, $\mathrm{Ma}$ is Mach number, and $\beta=\left(\mathrm{Ma}^{2}-1\right)^{1 / 2}$.

The flow goes against the lateral vibrations of the panel, so the nonconservative virtual work from aerodynamic load is negative and its expression is

$$
\delta W_{\mathrm{nc}}=-\int_{0}^{L} p(x, t) b \delta w(x, t) d x
$$

For a simply supported panel, the modal expansion of $w(x, t)$ can be assumed in the form

$$
w(x, t)=\sum_{n=1}^{\infty} \eta_{n}(t) \sin \left(\frac{n \pi x}{L}\right), \quad n=1,2,3, \ldots,
$$

where $n$ is the positive integer and $\eta_{n}(t)$ the generalized coordinate. After substituting (6) into all energy expressions, the system equations-of-motion are obtained via Lagrange's Equations

$$
\frac{d}{d t}\left(\frac{\partial T}{\partial \dot{q}}\right)-\frac{\partial T}{\partial q}+\frac{\partial V}{\partial q}+\frac{\partial D}{\partial \dot{q}}=Q_{\mathrm{nc}}
$$

with the generalized force $Q_{\mathrm{nc}}=\partial \delta W_{\mathrm{nc}} / \partial \delta q$ and generalized coordinates vector

$$
q(t)=\left[\begin{array}{llll}
\eta_{1}(t) & \eta_{2}(t) & \eta_{3}(t) & \cdots
\end{array}\right]^{T} .
$$

Finally, the system equations-of-motion are given as

$$
\begin{aligned}
& \mathbf{M} \ddot{q}(t)+\left(\mathbf{C}^{d}+\mathbf{C}^{a}\right) \dot{q}(t)+\left[\mathbf{K}^{e}+\mathbf{K}^{a}+\mathbf{K}^{P}(t)\right] q(t) \\
& \quad=0
\end{aligned}
$$


where the elements of coefficient matrices are

$$
\begin{aligned}
& \mathbf{M}_{m n}=\rho A \int_{0}^{L} \sin \left(\frac{m \pi x}{L}\right) \sin \left(\frac{n \pi x}{L}\right) d x, \\
& \mathbf{C}_{m n}^{d}=\xi E^{*} I \frac{m^{2} n^{2} \pi^{4}}{L^{4}} \int_{0}^{L} \sin \left(\frac{m \pi x}{L}\right) \sin \left(\frac{n \pi x}{L}\right) d x, \\
& \mathbf{C}_{m n}^{a} \\
& =\frac{2 b q_{0}}{\beta U_{0}} \frac{\left(\mathrm{Ma}^{2}-2\right)}{\left(\mathrm{Ma}^{2}-1\right)} \int_{0}^{L} \sin \left(\frac{m \pi x}{L}\right) \sin \left(\frac{n \pi x}{L}\right) d x, \\
& \mathbf{K}_{m n}^{e}=E^{*} I \frac{m^{2} n^{2} \pi^{4}}{L^{4}} \int_{0}^{L} \sin \left(\frac{m \pi x}{L}\right) \sin \left(\frac{n \pi x}{L}\right) d x, \\
& \mathbf{K}_{m n}^{a}=\frac{2 b q_{0}}{\beta} \frac{n \pi}{L} \int_{0}^{L} \sin \left(\frac{m \pi x}{L}\right) \cos \left(\frac{n \pi x}{L}\right) d x, \\
& \mathbf{K}_{m n}^{P}(t)=\frac{m n \pi^{2}}{L^{2}} P(t) \int_{0}^{L} \cos \left(\frac{m \pi x}{L}\right) \cos \left(\frac{n \pi x}{L}\right) d x .
\end{aligned}
$$

The stiffness matrix $\mathbf{K}^{P}(t)$ resulting from the periodic axial excitation introduces a periodically time-varying item into the system, so (9) is identified as a set of coupled Mathieu-Hill equations. Subsequently, the system equationsof-motion are transformed into the nondimensional (N.D.) form

$$
\begin{aligned}
\overline{\mathbf{M}}^{* *} \bar{q}(\tau)+\left(\overline{\mathbf{C}}^{d}+\overline{\mathbf{C}}^{a}\right)^{\frac{*}{q}}(\tau) \\
+\left[\overline{\mathbf{K}}^{e}+\overline{\mathbf{K}}^{a}+\overline{\mathbf{K}}^{P}(\tau)\right] \bar{q}(\tau)=0
\end{aligned}
$$

with the dimensionless parameters and coordinates:

$$
\begin{aligned}
\bar{x} & =\frac{x}{L}, \\
\bar{q} & =\frac{q(t)}{h}, \\
\Omega & =\frac{\pi^{2} h}{L^{2}} \sqrt{\frac{E^{*}}{12 \rho}}, \\
\tau & =t \Omega \\
\mu & =\frac{\rho_{0}}{\rho}, \\
\bar{\xi} & =\xi \Omega \\
P_{\mathrm{cr}} & =\frac{E^{*} I \pi^{2}}{L^{2}}, \\
f(\tau) & =\frac{P(t)}{P_{\mathrm{cr}}} \\
\sigma & =\frac{q_{0}}{E^{*}}, \\
\alpha & =\frac{h}{L},
\end{aligned}
$$

$$
\begin{aligned}
& \bar{\omega}=\frac{\omega}{\Omega}, \\
& \stackrel{(}{ }^{*}=\frac{d()}{d \tau} .
\end{aligned}
$$

The elements of the N.D. coefficient matrices in (11) are

$$
\begin{aligned}
\overline{\mathbf{M}}_{m n} & = \begin{cases}1 & \text { if } m=n \\
0 & \text { if } m \neq n,\end{cases} \\
\overline{\mathbf{C}}_{m n}^{d} & = \begin{cases}\bar{\xi} n^{4} & \text { if } m=n \\
0 & \text { if } m \neq n,\end{cases} \\
\overline{\mathbf{C}}_{m n}^{a} & = \begin{cases}\frac{2 \sqrt{6 \mu \sigma}\left(\mathrm{Ma}^{2}-2\right)}{\pi^{2} \alpha^{2}\left(\mathrm{Ma}^{2}-1\right)^{3 / 2}} & \text { if } m=n \\
0 & \text { if } m \neq n,\end{cases} \\
\overline{\mathbf{K}}_{m n}^{e} & = \begin{cases}n^{4} & \text { if } m=n \\
0 & \text { if } m \neq n,\end{cases} \\
\overline{\mathbf{K}}_{m n}^{a} & = \begin{cases}0 & \text { if } m=n \\
\frac{48 \sigma m n\left[1-(-1)^{m+n}\right]}{\sqrt{\mathrm{Ma}^{2}-1}\left(m^{2}-n^{2}\right) \pi^{4} \alpha^{3}} & \text { if } m \neq n,\end{cases} \\
\overline{\mathbf{K}}_{m n}^{P}(\tau) & = \begin{cases}n^{2} f(\tau) & \text { if } m=n \\
0 & \text { if } m \neq n .\end{cases}
\end{aligned}
$$

Here, $\overline{\mathbf{M}}, \overline{\mathbf{C}}^{d}, \overline{\mathbf{C}}^{a}$, and $\overline{\mathbf{K}}^{e}$ are constant symmetric matrices; $\overline{\mathbf{K}}^{a}$ is a constant skew-symmetric matrix; $\overline{\mathbf{K}}^{P}$ is a symmetric time-varying matrix with a period of $2 \pi / \bar{\omega}$.

\section{Mathematical Methods for Stability Analysis}

Due to the periodic axial excitations, (11) becomes a periodically linear time-varying system. Floquet theory is able to assess the stability of this type of systems through evaluating the eigenvalues of the Floquet transition matrix (FTM) numerically [24, 27-32]. The FTM method can obtain all unstable behaviors of a system but at the cost of intensively numerical computations, so the perturbation method originally developed by Hsu $[13,33]$ is modified in this paper to approximate the system stability boundary in an efficient way. The results from the perturbation (analytical) method will be compared with those from the FTM (numerical) method.

To implement Hsu's perturbation method, the timeinvariant part of the system stiffness matrix, $\overline{\mathbf{K}}^{e}+\overline{\mathbf{K}}^{a}$, needs to be diagonalized by its left and right eigenvectors [34]. The resulting equations-of-motion are

$$
\bar{q}(\tau)+\widetilde{\mathbf{C}} \bar{q}^{*}(\tau)+\left[\widetilde{\mathbf{K}}+\widetilde{\mathbf{K}}^{P}(\tau)\right] \bar{q}(\tau)=0
$$


with

$$
\begin{aligned}
\widetilde{\mathbf{C}} & =\mathbf{X}_{L}^{T}\left(\overline{\mathbf{C}}^{d}+\overline{\mathbf{C}}^{a}\right) \mathbf{X}_{R} \\
\widetilde{\mathbf{K}} & =\mathbf{X}_{L}^{T}\left(\overline{\mathbf{K}}^{e}+\overline{\mathbf{K}}^{a}\right) \mathbf{X}_{R} \\
\widetilde{\mathbf{K}}^{P}(\tau) & =\mathbf{X}_{L}^{T} \overline{\mathbf{K}}^{P}(\tau) \mathbf{X}_{R}, \\
\left(\overline{\mathbf{K}}^{e}+\overline{\mathbf{K}}^{a}\right) \mathbf{X}_{R} & =\lambda \mathbf{X}_{R} \\
\left(\overline{\mathbf{K}}^{e}+\overline{\mathbf{K}}^{a}\right)^{T} \mathbf{X}_{L} & =\lambda \mathbf{X}_{L} \\
\mathbf{X}_{L}^{T} \mathbf{X}_{R} & =\mathbf{I},
\end{aligned}
$$

where $\lambda$ is the eigenvalues of $\overline{\mathbf{K}}^{e}+\overline{\mathbf{K}}^{a}$ and $\mathbf{X}_{L}$ and $\mathbf{X}_{R}$ are the corresponding left and right eigenvectors, respectively, and orthonormal to each other. I is the identity matrix.

The damping matrix and the time-varying stiffness matrix resulting from the axial excitation are assumed to be small quantities relative to the time-invariant system stiffness for better predictability through Hsu's method. The standard form in Hsu's method is obtained by separating the regular and perturbed items in (14) and then expanding the periodic time-varying stiffness matrix into Fourier series,

$$
\bar{q}(\tau)+\widetilde{\mathbf{K}} \bar{q}(\tau)=-\widetilde{\mathbf{C}}_{\bar{q}}^{*}(\tau)-\widetilde{\mathbf{K}}^{P}(\tau) \bar{q}(\tau),
$$

where

$$
\widetilde{\mathbf{K}}^{P}(\tau)=\widetilde{\mathbf{K}}^{c} \cos (\bar{\omega} \tau)+\widetilde{\mathbf{K}}^{s} \sin (\bar{\omega} \tau) .
$$

With (16), the stability criteria given in $[13,33]$ can be applied by setting epsilon to one.

\section{Stability Boundaries for the Panel under Flow}

For the simple demonstrations of the model and the solving process developed above, only the first two modes are considered so that the closed-form stability solutions can be obtained. The axial excitation force considered here is a single frequency cosine function:

$$
\begin{aligned}
& \overline{\bar{q}}(\tau)+\left[\begin{array}{cc}
\omega_{1}^{2} & 0 \\
0 & \omega_{2}^{2}
\end{array}\right] \bar{q}(\tau) \\
& =-\left[\begin{array}{ll}
c_{11} & c_{12} \\
c_{21} & c_{22}
\end{array}\right] \frac{*}{\bar{q}}(\tau)-\left[\begin{array}{ll}
k_{11} & k_{12} \\
k_{21} & k_{22}
\end{array}\right] \cos (\bar{\omega} \tau) \bar{q}(\tau), \\
& f(\tau)=F \cos (\bar{\omega} \tau),
\end{aligned}
$$

where

$$
\begin{aligned}
& \omega_{1}=\sqrt{\frac{17}{2}-\frac{15}{2} \sqrt{1-\frac{\sigma^{2}}{\sigma_{c}^{2}}}}, \\
& \omega_{2}=\sqrt{\frac{17}{2}+\frac{15}{2} \sqrt{1-\frac{\sigma^{2}}{\sigma_{c}^{2}}}},
\end{aligned}
$$

$$
\begin{aligned}
c_{11}= & \bar{\xi}\left(\frac{17}{2}-\frac{15}{2} \frac{\sigma_{c}}{\sqrt{\sigma_{c}^{2}-\sigma^{2}}}\right) \\
& +\frac{2 \sqrt{6}\left(\mathrm{Ma}^{2}-2\right) \sqrt{\mu \sigma}}{\pi^{2} \alpha^{2}\left(\mathrm{Ma}^{2}-1\right)^{3 / 2}} \\
c_{22}= & \bar{\xi}\left(\frac{17}{2}+\frac{15}{2} \frac{\sigma_{c}}{\sqrt{\sigma_{c}^{2}-\sigma^{2}}}\right) \\
c_{12}= & \frac{15 \bar{\xi} \sigma}{2 \sqrt{\sigma_{c}^{2}-\sigma^{2}}}, \\
c_{21}= & -c_{12}, \\
k_{11}= & F\left(\frac{\left.\mathrm{Ma}^{2}-2\right) \sqrt{\mu \sigma}}{2}-\frac{3 \sigma_{c}^{2}}{2 \sqrt{\sigma_{c}^{2}-\sigma^{2}}}\right) \\
\sigma_{c}= & \frac{15}{128} \pi^{4} \alpha^{3} \sqrt{\mathrm{Ma}^{2}-1} \\
k_{22}= & F\left(\frac{5}{2}+\frac{3 \sigma_{c}}{2 \sqrt{\sigma_{c}^{2}-\sigma^{2}}}\right), \\
k_{21} \sqrt{\sigma_{c}^{2}-\sigma^{2}} & -k_{12}, \\
& \left(\frac{5}{2}\right)
\end{aligned}
$$

The material viscous loss factor is set to zero for the eigenanalyses in this paper. The stability boundaries can be obtained via solving the following equations:

1st principle resonance: $\bar{\omega}=2 \omega_{1}+\Delta \omega$

$$
\Delta \omega= \pm \sqrt{\frac{k_{11}^{2}}{4 \omega_{1}^{2}}-c_{11}^{2}}
$$

2nd principle resonance: $\bar{\omega}=2 \omega_{2}+\Delta \omega$

$$
\Delta \omega= \pm \sqrt{\frac{k_{22}^{2}}{4 \omega_{2}^{2}}-c_{22}^{2}}
$$

Combination resonance: $\bar{\omega}=\omega_{2}-\omega_{1}+\Delta \omega$

$$
\Delta \omega= \pm \sqrt{\frac{k_{12}^{2}}{4 \omega_{1} \omega_{2}}-c_{11} c_{22}} .
$$




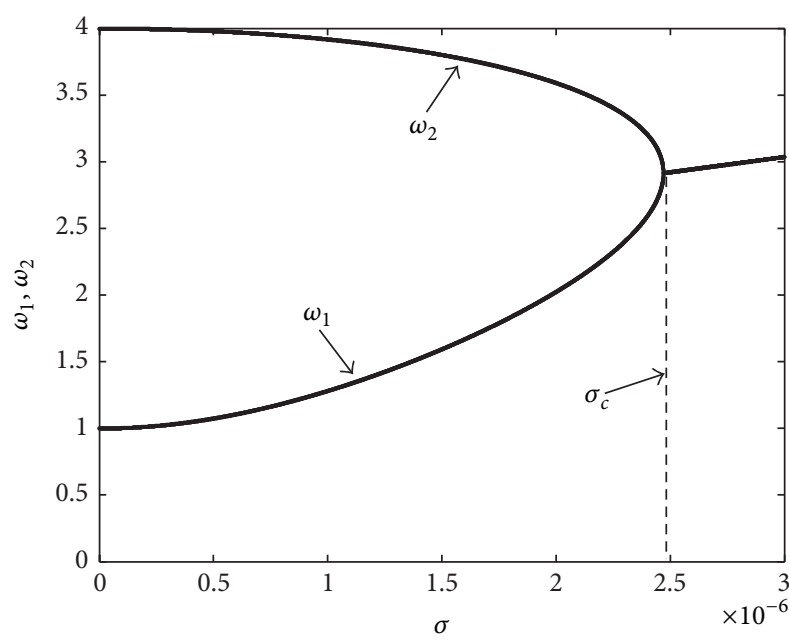

FIGURE 2: N.D. natural frequency variations with respect to dynamic pressure ratio: $\mathrm{Ma}=2, \alpha=0.005$, and $\sigma_{c}=2.47 \times 10^{-6}$.

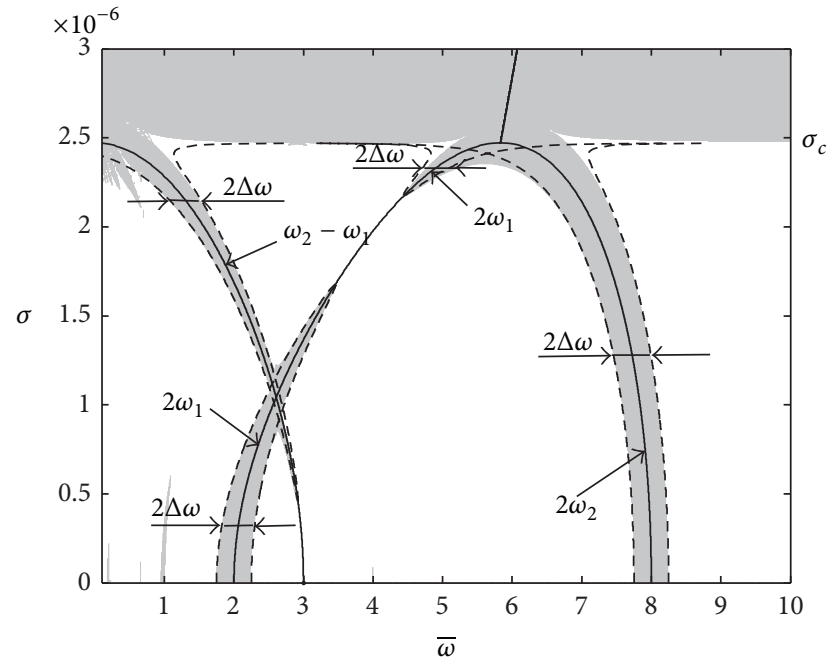

FIGURE 3: Stability plot for the flutter panel with respect to axial excitation frequency and dynamic pressure ratio: $\mathrm{Ma}=2, \alpha=0.005$, $\sigma_{c}=2.47 \times 10^{-6}, \mu=4.39 \times 10^{-5}$, and $F=0.5$.

\section{Numerical Results}

The N.D. natural frequencies due to materials and aerodynamic loads are plotted in Figure 2 with respect to the dynamic pressure ratio, $\sigma$. Once the dynamic pressure ratio exceeds the critical value that equals $\sigma_{c}$, two natural frequencies merge together, which means they are conjugate pairs and the system instability occurs.

The system stability with respect to the axial excitation frequency and the dynamic pressure ratio is shown in Figure 3. In this paper, the gray regions in stability plots indicate the instabilities computed by the numerical FTM method and the black dash lines are the stability boundaries calculated from the analytical perturbation method. The black solid lines in Figure 3 represent the N.D. natural frequencies. With the same observations as in Figure 2, the system flutters for the entire axial excitation frequency range calculated here

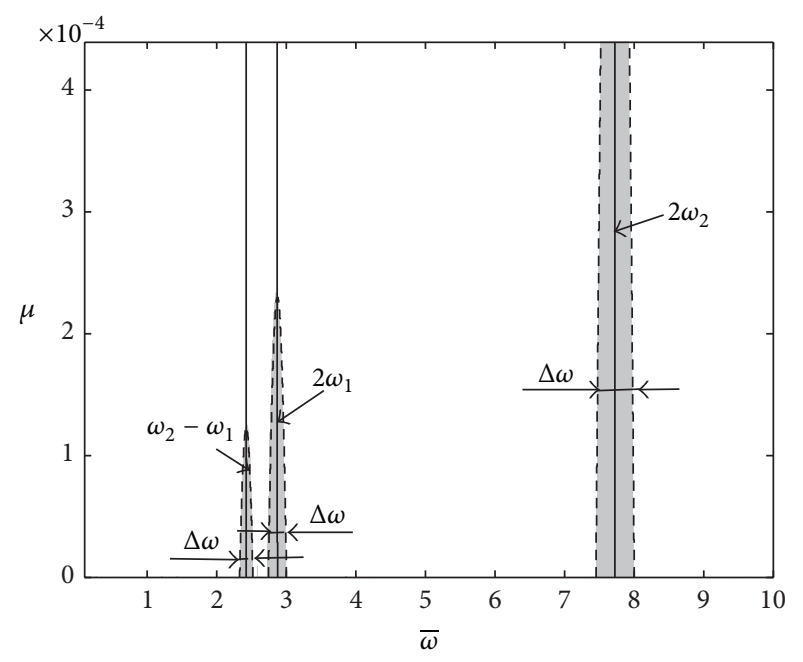

FIGURE 4: Stability plot for the flutter panel with respect to axial excitation frequency and air/panel density ratio: $\mathrm{Ma}=2, \alpha=0.005$, $\sigma_{c}=2.47 \times 10^{-6}, \sigma=1.27 \times 10^{-6}$, and $F=0.5$.

when the dynamic pressure ratio passes its critical value. The principle resonances and combination resonance given by the perturbation method successfully match those by the FTM method with a lot of computation savings.

The system stability with respect to the axial excitation frequency and the air/panel density ratio is shown in Figure 4 . Since $\sigma<\sigma_{c}$, the combination resonance and principal resonances are clearly separated. Again, the results from both the FTM method and the perturbation method match each other very well.

It can be observed in Figures 3 and 4 that the principal resonance of the second mode causes instabilities for all dynamic pressure ratio and air/panel density ratio values explored here. However, it could be stabilized by different axial excitation frequencies. The instabilities around the first principal resonance and combination resonance can be suppressed for some dynamic pressure ratio and air/panel density ratio values. Their axial excitation frequency stability boundaries are calculated by solving (20) for $\Delta \omega=0$ and the results are plotted in Figure 5. The system stability depends on both resonances in each area divided by those boundaries. The panel system is only stable when both resonances are stable, shown in the white area in Figure 5.

\section{Summary and Conclusions}

This paper investigates the parametric stability of the panel (beam) under both aerodynamic loads and axial excitations. The dimensionless equation-of-motion is derived, including material viscous damping, axial excitation, and aerodynamic load. The eigen-analyses based on the first two modes are taken as examples to explore the stability properties of the flutter panel system with an axial single frequency cosine excitation. Both numerical FTM method and analytical perturbation method solve the problem and their results match each other very well. 


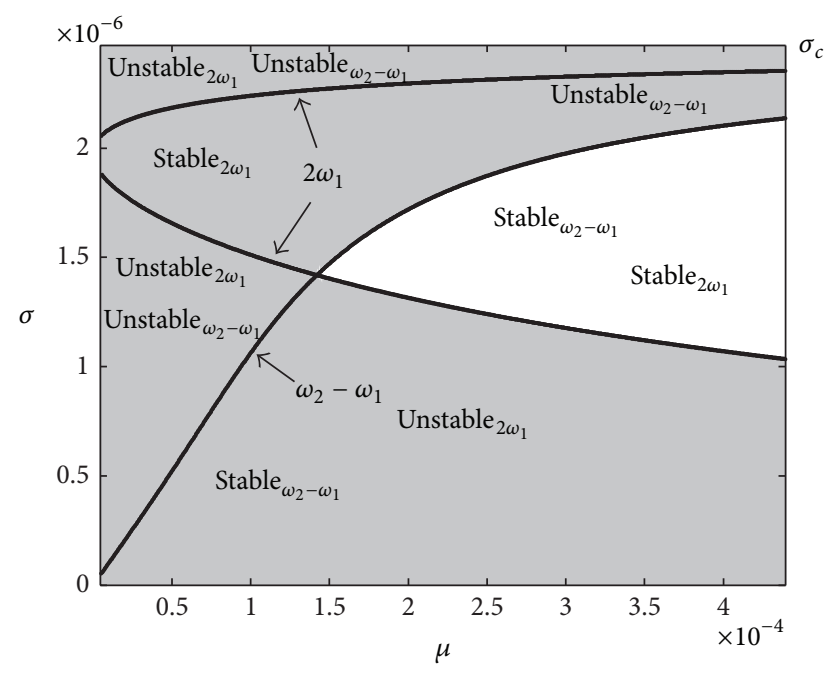

FIgURE 5: Stability plot for the flutter panel with respect to air/panel density ratio and dynamic pressure ratio: $\mathrm{Ma}=2, \alpha=0.005, \sigma_{c}=$ $2.47 \times 10^{-6}$, and $F=0.5$.

The panel may flutter under high-speed air flows when its out-of-plane dynamics couples with the aerodynamic loads. The parameter study was conducted for the system instability zones with respect to axial excitation frequency, air/panel density ratio, and air/panel dynamic pressure ratio. Different from the static axial force, this paper introduces a periodic axial excitation that brings the system into the timevarying domain. The axial excitation force could increase the panel stiffness locally to overcome aerodynamic loads when interacting with the out-of-plane vibrations. The study results in this paper indicate that the system is stable under the combinations of the proper excitation frequency and certain air/panel density ratio and dynamic pressure ratio.

The perturbation method developed in this paper saves lots of computations, which can help understand the flutter phenomenon of the panel with axial excitations more efficiently.

\section{Competing Interests}

The authors declare that they have no competing interests.

\section{References}

[1] F. Liu, J. Cai, Y. Zhu, H. M. Tsai, and A. S. F. Wong, "Calculation of wing flutter by a coupled fluid-structure method," Journal of Aircraft, vol. 38, no. 2, pp. 334-342, 2001.

[2] J. L. Hall, "A review of ballute technology for planetary aerocapture," in Proceedings of the 4th IAA Conference on Low Cost Planetary Missions, pp. 1-10, Laurel, Md, USA, May 2000.

[3] H. C. Nelson and H. J. Cunningham, "Theoretical investigation of flutter of two-dimensional flat panels with one surface exposed to supersonic potential flow," NACA Technical Note 3465/Report 1280, 1955.

[4] M. D. Olson, "Finite elements applied to panel flutter," AIAA Journal, vol. 5, no. 12, pp. 2267-2270, 1967.
[5] P. C. Parks, "A stability criterion for panel flutter via the second method of Liapunov," AIAA Journal, vol. 4, no. 1, pp. 175-177, 1966.

[6] J. Dugundji, "Theoretical considerations of panel flutter at high supersonic Mach numbers., AIAA Journal, vol. 4, no. 7, pp. 1257-1266, 1966.

[7] E. H. Dowell, "Nonlinear oscillations of a fluttering plate," AIAA Journal, vol. 4, no. 7, pp. 1267-1275, 1966.

[8] E. H. Dowell, "Nonlinear oscillations of a fluttering plate II," AIAA Journal, vol. 5, no. 10, pp. 1856-1862, 1967.

[9] R. C. Zhou, C. Mei, and J.-K. Huang, "Suppression of nonlinear panel flutter at supersonic speeds and elevated temperatures," AIAA Journal, vol. 34, no. 2, pp. 347-354, 1996.

[10] D. J. Gee, "Numerical continuation applied to panel flutter," Nonlinear Dynamics, vol. 22, no. 3, pp. 271-280, 2000.

[11] S. Tizzi, "Influence of non-linear forces on beam behaviour in flutter conditions," Journal of Sound and Vibration, vol. 267, no. 2, pp. 279-299, 2003.

[12] T. Iwatsubo, Y. Sugiyama, and S. Ogino, "Simple and combination resonances of columns under periodic axial loads," Journal of Sound and Vibration, vol. 33, no. 2, pp. 211-221, 1974.

[13] C. S. Hsu, "On the parametric excitation of a dynamic system having multiple degrees of freedom," ASME Journal of Applied Mechanics, vol. 30, pp. 367-372, 1963.

[14] S. K. Sinha, "Dynamic stability of a Timoshenko beam subjected to an oscillating axial force," Journal of Sound and Vibration, vol. 131, no. 3, pp. 509-514, 1989.

[15] S. K. Sahu and P. K. Datta, "Parametric instability of doubly curved panels subjected to non-uniform harmonic loading," Journal of Sound and Vibration, vol. 240, no. 1, pp. 117-129, 2001.

[16] S. K. Sahu and P. K. Datta, "Dynamic stability of curved panels with cutouts," Journal of Sound and Vibration, vol. 251, no. 4, pp. 683-696, 2002.

[17] M. Ganapathi, B. P. Patel, P. Boisse, and M. Touratier, "Nonlinear dynamic stability characteristics of elastic plates subjected to periodic in-plane load," International Journal of NonLinear Mechanics, vol. 35, no. 3, pp. 467-480, 2000.

[18] K. Nagai and N. Yamaki, "Dynamic stability of circular cylindrical shells under periodic compressive forces," Journal of Sound and Vibration, vol. 58, no. 3, pp. 425-441, 1978.

[19] C. Massalas, A. Dalamangas, and G. Tzivanidis, "Dynamic instability of truncated conical shells, with variable modulus of elasticity, under periodic compressive forces," Journal of Sound and Vibration, vol. 79, no. 4, pp. 519-528, 1981.

[20] V. B. Kovtunov, "Dynamic stability and nonlinear parametric vibration of cylindrical shells," Computers \& Structures, vol. 46, no. 1, pp. 149-156, 1993.

[21] K. Y. Lam and T. Y. Ng, "Dynamic stability of cylindrical shells subjected to conservative periodic axial loads using different shell theories," Journal of Sound and Vibration, vol. 207, no. 4, pp. 497-520, 1997.

[22] T. Y. Ng, K. Y. Lam, K. M. Liew, and J. N. Reddy, "Dynamic stability analysis of functionally graded cylindrical shells under periodic axial loading," International Journal of Solids and Structures, vol. 38, no. 8, pp. 1295-1309, 2001.

[23] F. Pellicano and M. Amabili, "Stability and vibration of empty and fluid-filled circular cylindrical shells under static and periodic axial loads," International Journal of Solids and Structures, vol. 40, no. 13-14, pp. 3229-3251, 2003.

[24] M. Ruzzene, "Dynamic buckling of periodically stiffened shells: application to supercavitating vehicles," International Journal of Solids and Structures, vol. 41, no. 3-4, pp. 1039-1059, 2004. 
[25] P. Hagedorn and L. R. Koval, "On the parametric stability of a Timoshenko beam subjected to a periodic axial load," IngenieurArchiv, vol. 40, no. 3, pp. 211-220, 1971.

[26] K.-J. Yang, K.-S. Hong, and F. Matsuno, "Robust adaptive boundary control of an axially moving string under a spatiotemporally varying tension," Journal of Sound and Vibration, vol. 273, no. 4-5, pp. 1007-1029, 2004.

[27] Z. Viderman, F. P. J. Rimrott, and W. L. Cleghorn, "Parametrically excited linear nonconservative gyroscopic systems," Mechanics of Structures and Machines, vol. 22, no. 1, pp. 1-20, 1994.

[28] V. V. Bolotin, The Dynamic Stability of Elastic Systems, HoldenDay, 1964.

[29] C. S. Hsu, "On approximating a general linear periodic system," Journal of Mathematical Analysis and Applications, vol. 45, pp. 234-251, 1974.

[30] P. P. Friedmann, "Numerical methods for determining the stability and response of periodic systems with applications to helicopter rotor dynamics and aeroelasticity," Computers and Mathematics with Applications, vol. 12, no. 1, pp. 131-148, 1986.

[31] O. A. Bauchau and Y. G. Nikishkov, "An implicit Floquet analysis for rotorcraft stability evaluation," Journal of the American Helicopter Society, vol. 46, no. 3, pp. 200-209, 2001.

[32] H. A. DeSmidt, K. W. Wang, and E. C. Smith, "Coupled torsionlateral stability of a shaft-disk system driven throuoh a universal joint," Journal of Applied Mechanics, Transactions ASME, vol. 69, no. 3, pp. 261-273, 2002.

[33] C. S. Hsu, "Further results on parametric excitation of a dynamic system," ASME Journal of Applied Mechanics, vol. 32, pp. 373-377, 1965.

[34] L. Meirovitch, Computational Methods in Structural Dynamics, Sijthoff \& Noordhoff, 1980. 


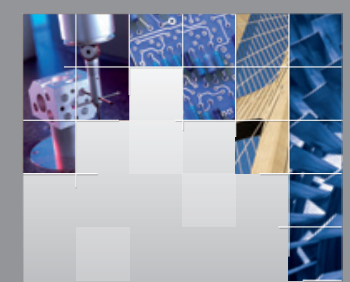

\section{Enfincering}
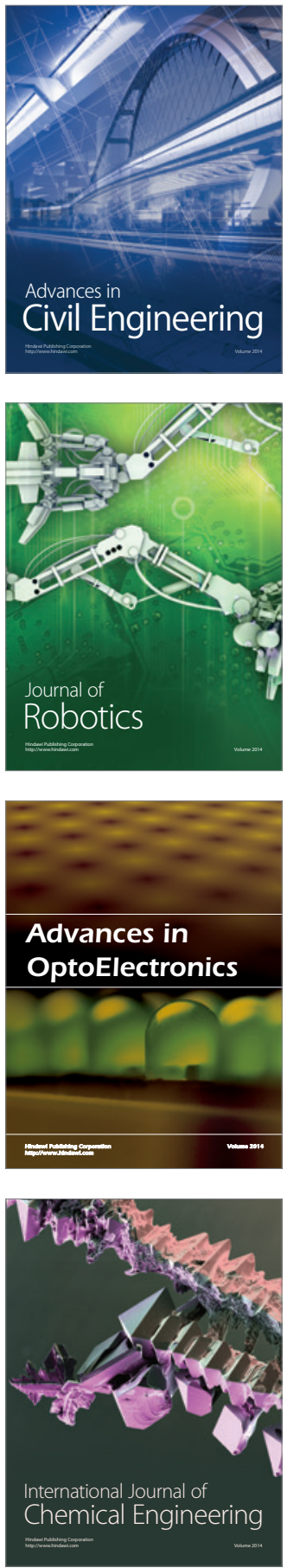

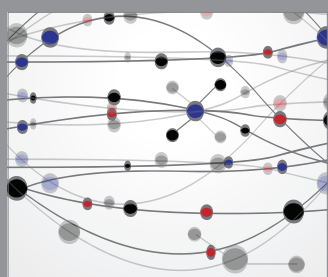

The Scientific World Journal

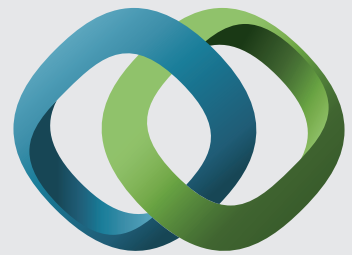

\section{Hindawi}

Submit your manuscripts at

http://www.hindawi.com
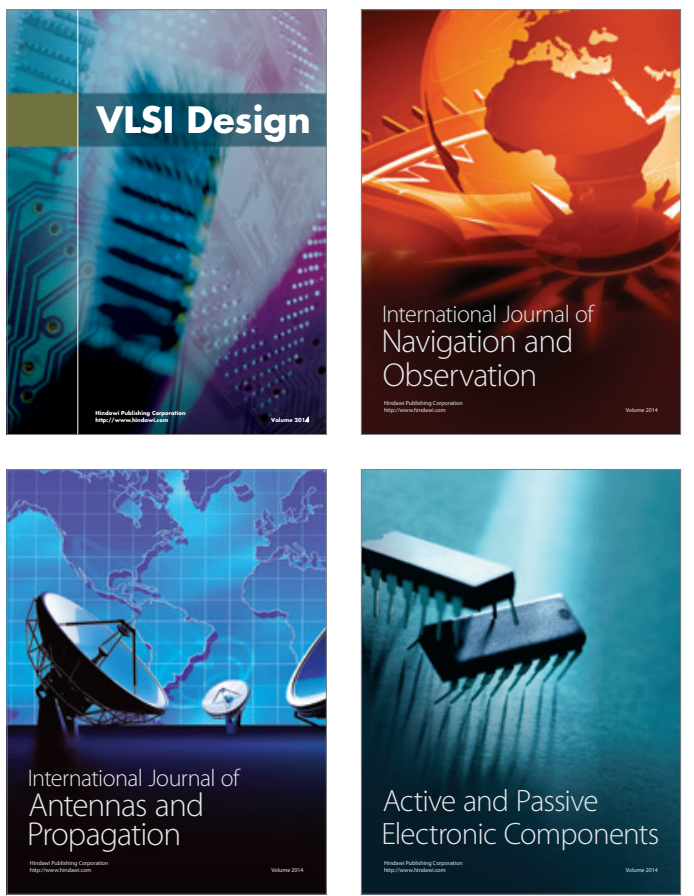
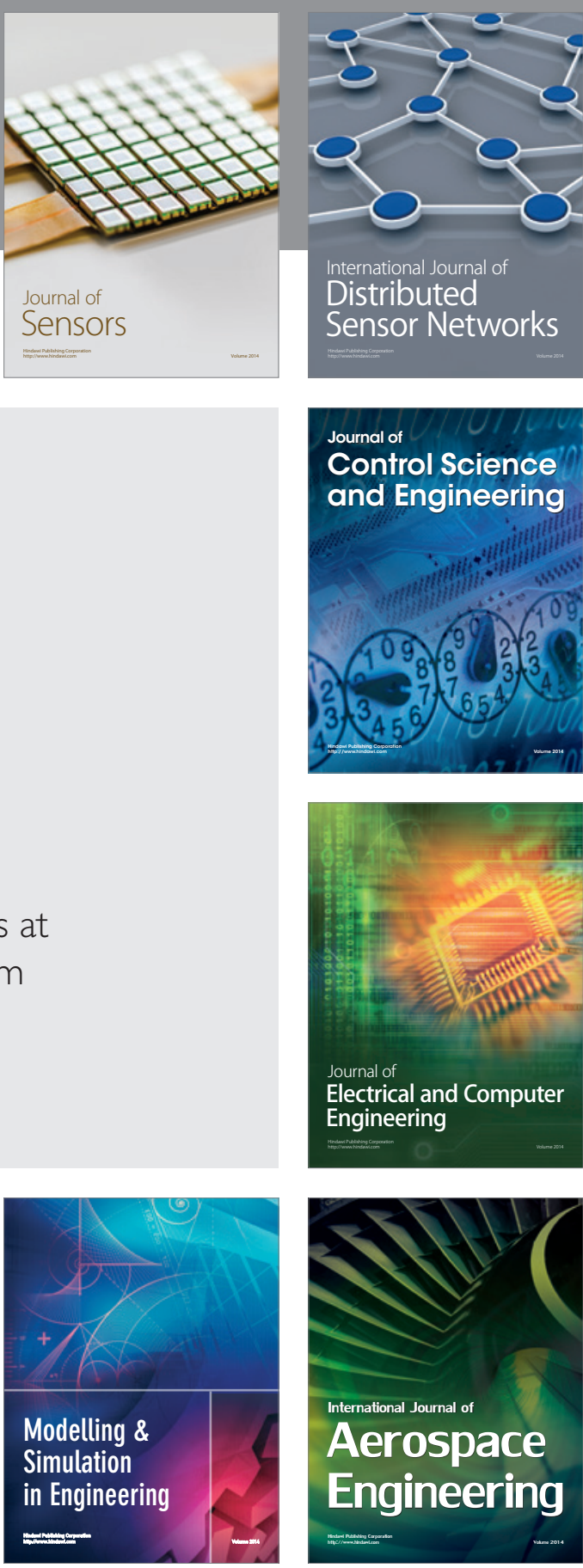

International Journal of

Distributed

Sensor Networks

Journal of

Control Science

and Engineering
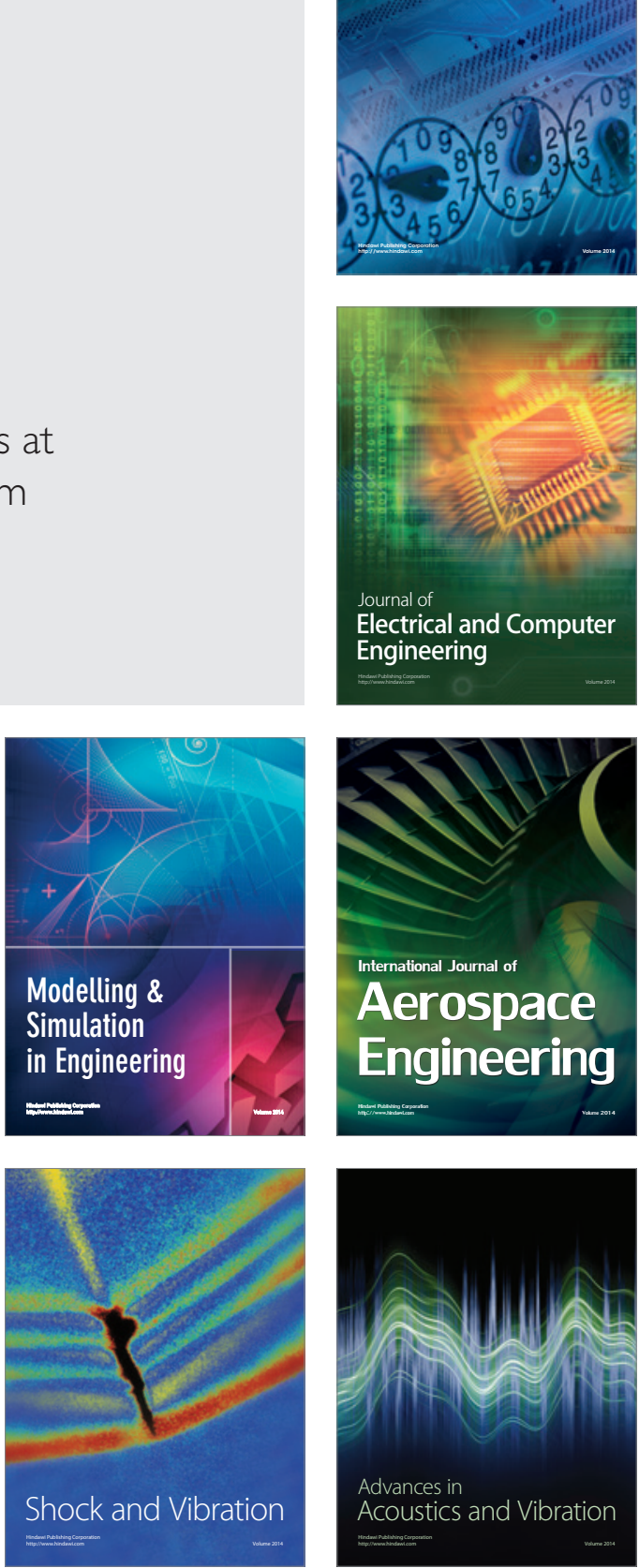